\title{
Diacronie
}

Studi di Storia Contemporanea

$N^{\circ} 16,4 \mid 2013$

Le monarchie nell'età dei nazionalismi

\section{I re soldati e la Nazione}

L'esercito come strumento di legittimazione della monarchia sabauda 1848-1900

Jacopo Lorenzini

\section{(2) OpenEdition}

Journals

\section{Edizione digitale}

URL: http://journals.openedition.org/diacronie/890

DOI: 10.4000/diacronie.890

ISSN: 2038-0925

Editore

Association culturelle Diacronie

Notizia bibliografica digitale

Jacopo Lorenzini, «I re soldati e la Nazione », Diacronie [Online], № 16, 4 | 2013, documento 6, Messo online il 01 décembre 2013, consultato il 20 avril 2019. URL : http://journals.openedition.org/ diacronie/890 ; DOI : 10.4000/diacronie.890 


\section{Diacronie}

\section{6/}

\section{I re soldati e la Nazione}

\section{L'esercito come strumento di legittimazione della monarchia sabauda 1848-190o}

Jacopo LORENZINI *

Le forze armate, $e$ in particolare l'esercito a coscrizione obbligatoria, furono un potente strumento di nazionalizzazione delle masse. Nel caso italiano, la monarchia sabauda comprese rapidamente di dover cambiare il proprio paradigma da dinastico a nazionale, per mantenere l'egemonia sul processo unitario e battere l'opzione democratica rappresentata da Garibaldi e dai suoi volontari. D'altra parte, il corpo ufficiali necessitava di un punto di riferimento in grado di mettere in rilievo l'importanza dell'esercito durante la fase che condusse l'Armata Sarda a divenire Esercito italiano. Si venne così a creare un rapporto di reciproca legittimazione tra monarchia ed esercito nell'ambito della costruzione di un'identità italiana unitaria e nazionale.

\section{Introduzione}

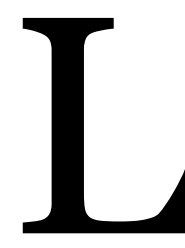

o si è scritto centinaia, migliaia di volte: l'esercito a coscrizione universale fu uno dei due strumenti principali, assieme all'istruzione primaria, coinvolti nel XIX secolo nei processi di nation building e nella creazione di comunità nazionali culturali, politiche ed emozionali soprattutto nel caso di quella italiana, neonata e strutturalmente divisa. Tuttavia lo studio di come l'istituzione militare agisse nella pratica quotidiana - in base a quale ideologia, governata da quale élite socio-professionale - e di quale rapporto infine la legasse a quello che ne viene universalmente indicato come il vertice - l'istituzione monarchica - è ancora oggetto in 
Italia di rari, sparsi contributi $^{1}$, causando una persistente confusione in sede storiografica quando si tratta di inserire la variabile militare in un quadro interpretativo dell'Italia moderna e contemporanea. In questa sede ci occuperemo dell'ultimo tra gli aspetti elencati, esaminando anzitutto quale fosse la natura del rapporto tra monarchia ed esercito, quali criticità presentasse, quali strumenti la monarchia mettesse in campo per garantirsi una fedeltà che la storiografia vuole scevra di dubbi, ma che in alcuni momenti della storia unitaria scricchiola non poco. Entreremo poi nel vivo dei processi di legittimazione attivatisi all'indomani dell'Unità, ma di fatto iniziati già a partire dal 1848-1849. Processi nei quali ideologia della vita militare, immagine del sovrano ed esibizione pubblica della forza armata contribuiscono a restituirci un esercito come pilastro non solo pratico-repressivo, ma anche e soprattutto simbolico dell’Unità nazionale sotto la monarchia sabauda.

\section{Monarchia ed esercito}

\subsection{La prerogativa regia}

I Savoia protagonisti dell'Unità nazionale non sono nuovi a imprese di omogeneizzazione sotto il simbolo regio attraverso lo strumento militare: quel che riuscirono a fare tra 1714 (quando Vittorio Amedeo II ricevette la corona reale) e 1796 (quando la quadriennale resistenza piemontese cedette alle armate della rivoluzione guidate dal giovane Bonaparte) è per alcuni versi premonitore di quanto accadrà prima e dopo il 1861. In un secolo di «feroce assolutismo regio»² la casa di Savoia riesce a fondare uno stato nazionale a tutti gli effetti (seppur d'Ancien Régime), utilizzando l'istituzione militare come uno dei più potenti mezzi a sua disposizione - non a caso, accanto all'organizzazione di istruzione e cultura. Riduzione del reclutamento estero bilanciata dalla creazione di reggimenti provinciali, istituzione di accademie e collegi militari, cooptazione delle nobiltà locali nel corpo ufficiali sono altrettanti elementi che contribuiscono a fare del Regno di Sardegna uno stato-nazione in senso pienamente moderno. Non sono casuali le parole scritte dal marchese di Bersezio (esponente di

\footnotetext{
${ }^{1}$ Per un quadro della storiografia militare italiana, e per una rassegna delle sue persistenti lacune, rimandiamo a LABANCA, Nicola (a cura di), Storie di guerre e di eserciti. Gli studi italiani di storia militare negli ultimi venticinque anni, Milano, Unicopli, 2011.

2 FERRONE, Vincenzo, «Un Re, un esercito, una nazione; il riarmo italiano nel settecento tra innovazioni tecnologiche, assolutismo e identità nazionali d'Antico Regime», in BARBERIS, Walter (a cura di), Storia d'Italia, Annali XVIII. Guerra e pace, Torino, Einaudi, 2002, pp. 383414 .
} 
quella internazionale aristocratica delle armi descritta da Piero Del Negro3) proprio verso la fine del secolo: «les prussiens font ceci, les hannoveriens font cela, les autrichiens ont telles manoeuvres, les françois telles autres. Mais nous ne sommes aucune de ces nations. Nous sommes piémontois, voyons d'abord ce qui convient à des piémontois»4.

Dall'importanza dello strumento militare per la dinastia trae origine la storia della cosiddetta prerogativa regia, articolo 5 dello Statuto Albertino (1848):

\begin{abstract}
Al Re solo appartiene il potere esecutivo. Egli è il Capo Supremo dello Stato; comanda tutte le forze di terra e di mare; dichiara la guerra; fa i trattati di pace, d'alleanza, di commercio ed altri, dandone notizia alle Camere tosto che l'interesse e la sicurezza dello Stato il permettano, ed unendovi le comunicazioni opportune5.
\end{abstract}

Ispirato ad aspetti similari delle carte costituzionali belga (1831) e francesi del 1814 e 1830, oltre che alle istituzioni inglesi, l'articolo 5 rimane pienamente operativo ben oltre i suoi omologhi europei ${ }^{6}$, tanto da essere utilizzato per sancire l'entrata dell'Italia nella Grande Guerra, e da costituire il principale pomo della discordia tra monarchia e fascismo. Per quale motivo la parlamentarizzazione delle istituzioni politiche italiane, effettiva e riconosciuta già pochi anni dopo l'unità, non agisce su questo aspetto?

Contrariamente a quanti sostengono che siano le sconfitte del 1848-1849 a portare il Piemonte - e poi l'Italia - dal regime costituzionale puro previsto dallo Statuto a quello parlamentare 7 , riteniamo che proprio dal 1849 e dalla disponibilità di Vittorio Emanuele e Cavour a legare, ancora più di quanto avesse fatto Carlo Alberto, causa dinastica e causa nazionale, nasca un compromesso che definisce sfere di competenza parlamentari e sovrane, cristallizzandole. Si ha dunque una parlamentarizzazione delle istituzioni che non sfiora la prerogativa regia.

Secondo Lucio $\mathrm{Ceva}^{8}$, sistemi politici mobili come quelli delle altre monarchie costituzionali europee (che permettono ed anzi prevedono un'alternanza al potere di

3 Cfr. DEL NEGRO, Piero, Guerra ed eserciti da Machiavelli a Napoleone, Roma-Bari, Laterza, 2001.

${ }_{4}^{4}$ Cit. in FERRONE, Vincenzo, op. cit., p. 407.

5 Statuto Albertino, 4 marzo 1848 ,

URL: < http://www.quirinale.it/qrnw/statico/costituzione/statutoalbertino.htm > [consultato il 28 dicembre 2013].

${ }^{6}$ Anche se, nel caso belga, i sovrani se ne avvalgono tanto nel 1914 che nel 1940 per assumere il comando delle forze armate in guerra.

7 Opinione di H. McGaw Smyth, cit. in DE LEONARDIS, Massimo, «Monarchia, famiglia reale e forze armate nell'Italia unita», in Rassegna Storica del Risorgimento, 86, 2/1999, pp. 77-102, p. 184.

${ }^{8}$ CEVA, Lucio, «Monarchia e militari dal Risorgimento alla Grande Guerra (1848-1915)», in Nuova Antologia, 2197, 1/1996, pp. 112-136. 
partiti differenti) riducono il margine di manovra della Corona, fattore al quale si aggiunge nel caso britannico l'assenza di un esercito permanente a coscrizione universale. Nell'Italia del connubio, che quell'alternanza blocca per anni, se i conservatori cavouriani si disinteressano della questione, la sinistra moderata rinuncia a metterla in discussione in cambio della scelta in senso nazionale della monarchia. Scelta sancita in ambito militare dalla modifica nel giugno 1848 del giuramento: al vecchio «giuro solennemente d'essere fedele a Dio, ed alla Maestà del Re nostro signore [...] pel sostegno della sua Corona e della piena sua Autorità Sovrana» viene sia tolto il riferimento a Dio che aggiunta la frase «osservare lealmente lo statuto e le leggi dello Stato». Il conseguente proclama che Carlo Alberto rivolge alle truppe è stato interpretato sia come una dichiarazione d'identità tra monarchia e causa nazionale ${ }^{9}$ che come una sottolineatura della prerogativa regia in materia militare - a nostro parere questioni per nulla antitetiche, anzi. In altre parole, purché la monarchia diventi e si mantenga guida del movimento nazionale, e porti in dote alla causa lo strumento militare, il parlamento del connubio è disposto a lasciarle margine di manovra autonoma in campo militare e diplomatico. Dopo gli anni della Destra, impegnata nel pareggio di bilancio e nella situazione emergenziale della lotta al brigantaggio, e l'avvento della Sinistra Storica, la staticità del sistema politico è perpetuata dall'uso di «basare il potere su alleanze mutevoli all'interno di un'amorfa maggioranza parlamentare piuttosto che su un singolo partito con un programma ben definito e coerente ${ }^{10}$. In altre parole i governi del trasformismo, troppo deboli politicamente per porsi in contrasto con la Corona, lasciano immutata la prerogativa regia. In seguito, la risoluzione parlamentare del conflitto tra risorgimento monarchico e democratico (a tutto favore del primo) impersonata da Francesco Crispi rende superati eventuali conflitti istituzionali a proposito della prerogativa.

Il conflitto tra Trono e Altare impedisce inoltre la formazione in Italia di un blocco conservatore di maggioranza, blocco la cui presenza favorisce invece il mantenimento della forma costituzionale pura in Germania ${ }^{11}$. Tra l'altro, la destra italiana di fine secolo, quella che invoca il Torniamo allo Statuto, vuole un Re "capo di cittadinanza" e non "capo di bande e di truppe": «Le dinastie devono smettere l'apparenza come la realtà, di essere venute fuori dalla guerra, e intese soprattutto a questa» ${ }^{12}$. Posizione che

\footnotetext{
9 CEVA, Lucio, «Costituzione e funzionamento del comando dell'esercito dal 1918 al 1943», in Il problema dell'alto comando dell'esercito italiano dal Risorgimento al Patto Atlantico, atti di convegno, Roma 1985, pp. 177-178.

${ }^{10}$ CEVA, Lucio, «Monarchia e militari dal Risorgimento alla Grande Guerra (1848-1915)», cit., p. 135 .

${ }^{11}$ DE LEONARDIS, Massimo, op. cit., p. 185.

${ }_{12}$ Articolo di Ruggero Bonghi del 1893 (BONGHI, Ruggero, L'Ufficio del principe in uno Stato
} 
cozza sia con la gelosia sabauda per la prerogativa ${ }^{13}$, sia con la politica di identificazione tra Casa di Savoia e Nazione italiana attraverso la tradizione guerriera, e che costituisce a nostro parere uno dei motivi per i quali Umberto I non cede alle sirene sonniniane. Da notare anche come l'esistenza, mai seriamente insidiata, della prerogativa abbia evitato conflitti costituzionali paragonabili a quelli avvenuti nell'impero tedesco: il contrasto tra Corona e parlamento in materia militare si concretizza semmai nell'aumento delle prerogative del capo di stato maggiore a discapito del ministro della Guerra ai primi del Novecento ${ }^{14}$.

Lucio Ceva scrive che «la sopravvivenza della prerogativa regalava alla corona uno strumento di cui essa non volle o non seppe mai fare un uso nazionalmente creativo, neppure nel suo interesse. Poteva essere un'utile "stanza di compensazione" tra poteri separati. Rimase solo strumento di intrigo o territorio d'inerzia» ${ }^{15}$. Vedremo invece come la prerogativa regia, che lega monarca ed esercito senza mediazioni di sorta, sia alla base del "contratto" che permette di utilizzare quest'ultimo sia in senso conservativo dell'istituzione monarchica, che di nazionalizzazione del popolo italiano.

\subsection{Conflitto di modelli}

La questione della prerogativa non è solo riferita al comando in guerra e al suo esercizio in senso puramente politico-istituzionale: la prerogativa è funzionale a mantenere in tempo di pace quel grip sul paese che in periodo di guerra si ottiene con lo stato d'emergenza e il comando delle operazioni; grip che i Savoia decidono di conseguire sposando l'idea nazionale. Ergo prerogativa regia in campo militare e legittimazione nazionale sono strettamente connesse, ma occorre che l'esercito sia fedele alla monarchia.

Si è sempre detto di come la Casa di Savoia goda di «incondizionato lealismo monarchico» da parte delle forze armate e in particolare del corpo ufficiali, i quali percepiscono «la Corona come la fonte stessa della [loro] esistenza» ${ }^{16}$. Di fatto la questione è ben più complessa, e si configura a nostro parere come un rapporto di

libero) cit. ibidem, p. 186.

${ }^{13}$ Secondo Luigi Cadorna, la sua mancata nomina a capo di stato maggiore ancora nel 1908 è causata dai suoi pur cautissimi suggerimenti sulla sottrazione al sovrano del comando effettivo in guerra. Ibidem, p. 131.

14 Aumento che non ci appare particolarmente decisivo per l'equilibrio delle forze in campo, considerato che la grandissima maggioranza dei ministri militari sono graditi al sovrano, quando non direttamente designati dallo stesso (v. infra, 1.3).

${ }_{15}$ CEVA, Lucio, « Monarchia e militari dal Risorgimento alla Grande Guerra (1848-1915)», op. cit., p. 136.

${ }^{16}$ CEVA, Lucio, Storia delle forze armate in Italia, Torino, UTET, 1999, pp. 64-65. 
mutua interdipendenza e legittimazione.

Ad unità conseguita ormai da oltre vent'anni, Umberto I confida a Domenico Farini come la sua massima preoccupazione sia che non si riduca «l'esercito sul modello borbonico, pagnottante, pronto a servire il re, Garibaldi, la Repubblica, il Papa: questo è il fine recondito della democrazia» ${ }^{17}$. L'esercito non deve servire altri che il sovrano, e forse Umberto ricorda il conflitto di modelli militari e socio-istituzionali che si era delineato negli anni dell'Unità.

Tutto il Risorgimento è percorso da differenti concezioni sia della guerra, che dell'istituzione militare. $\mathrm{Si}$ va dalla guerra partigiana o per bande teorizzate rispettivamente da Bianco di Saint-Jorioz e Mazzini, alla rivoluzione militare e sociale di Pisacane, alle molteplici esperienze volontaristiche culminate nell'avventura garibaldina, che finisce per offuscare tutte le precedenti. Agli antipodi c'è l'Armata Sarda, l'esercito di caserma costituito da professionisti delle armi e inquadrato da ufficiali di carriera tutto fuorché democratici. Al momento dell'unità, quando si tratta di creare un esercito nazionale unitario, sono questi i due modelli che si scontrano; e non è uno scontro esclusivamente sul piano tecnico-militare, tutt'altro. L'eventuale mantenimento dell'esercito garibaldino, «anticipazione o [...] metonimia della nazione in armi, preludio alla formazione di un esercito di popolo» ${ }^{18}$ appare a molti come un punto decisivo a favore dell'opzione democratica, una minaccia sia per la monarchia che per il suo esercito e, di conseguenza, per l'appena raggiunta unità nazionale. Dall'altra parte, un esercito regolare che nel 1848 «era disposto a battersi per il proprio re, ma non altrettanto per l'indipendenza italiana [e nel quale] la situazione del 1859 era già migliore, ma non quanto si è detto e scritto» ${ }^{19}$. Insomma, se anche buona parte dei garibaldini sono disposti a riconoscere l'autorità regia, i politici liberali temono la sovversione sociale, e gli ufficiali sardi la corruzione dello spirito militare sabaudo basato sul ferreo pilastro della disciplina e della fedeltà dinastica.

Questo rende un compromesso tra i due modelli di fatto impraticabile, e il conflitto

\footnotetext{
${ }_{17}$ Cit. in LABANCA, Nicola, «Militari deputati e deputati militari (1848-1922)», in DEL NEGRO, Piero, CAFORIO, Giuseppe (a cura di), Ufficiali e Società; interpretazioni e modelli, Milano, Angeli, 1988, p. 444.

18 BANTI, Alberto Mario, MONDINI, Marco, Da Novara a Custoza: culture militari e discorso nazionale tra Risorgimento e Unità, in BARBERIS, Walter (a cura di), Storia d'Italia, Annali XVIII, Torino, Einaudi, 2002, pp. 417-462, citazione da p. 428.

19 MAZZETTI, Massimo, «Dagli eserciti preunitari all'esercito italiano», in Rassegna storica del Risorgimento, 4/1972, pp. 563-592, citazione da p. 574; anche il fratello di re Vittorio Emanuele II, Ferdinando, scriveva nel 1849 che gli ufficiali piemontesi si erano trovati «a combattere per una causa [quella nazionale] la quale era grande e generosa, se si vuole, ma affatto opposta a tutti i principi in cui [erano] stati allevati», cit. in DEL NEGRO, Piero, LABANCA, Nicola, STADERINI, Alessandra (a cura di), Militarizzazione e nazionalizzazione nella storia d'Italia, Milano, Unicopli, 2005, p. 17.
} 
si risolve con la brutale vittoria dell'esercito regolare, appena moderata da una integrazione, parziale e lenta, dei volontari. ${ }^{20}$ Come nel secolo precedente, ma con una differenza fondamentale: non si tratta più di usare l'istituzione militare per omogeneizzare realtà provinciali in uno stato moderno, bensì realtà regionali (e compiutamente statuali, perlomeno nel caso delle Due Sicilie) in una comunità nazionale - oltre che di fare i conti con la liquidata, ma ancora viva almeno nei timori dell'establishment liberal-monarchico, opzione democratica.

Differenza che non sfugge né alla monarchia né ai vertici delle neonate forze armate unitarie, che si trovano di fronte un doppio problema: scongiurare un rigurgito democratico, soprattutto all'interno dell'esercito che ha incorporato parte dei volontari, e rendere lo stesso esercito uno strumento efficace per l'omogeneizzazione in senso nazionale del neonato Regno d'Italia. Alla vittoria sull'opzione democratica si affiancano quindi alcuni tratti del nuovo esercito che, almeno nelle intenzioni di Manfredo Fanti, primo suo responsabile e, significativamente, ufficiale piemontesizzato ma proveniente da una lontana militanza volontaria, devono concorrere alla sua nazionalizzazione. In quest'ottica sono inquadrabili, ad esempio, la scelta del reclutamento nazionale e non territoriale, la rotazione delle sedi dei reparti (finalizzata a far fisicamente conoscere altre zone del paese a ufficiali - in gran parte piemontesi e coscritti), l'estensione dell'istituto della Guardia Nazionale a tutto il territorio del Regno. Non intendiamo negare le finalità pratiche soprattutto dei primi due elementi (in particolare la possibilità di utilizzare le truppe per finalità di ordine pubblico senza temere defezioni a favore di compaesani), ma riteniamo sincere sia le preoccupazioni per la tenuta della neonata unità in caso di reclutamento territoriale, sia la volontà da parte perlomeno di alcuni tra i primi vertici dell'esercito di fare dello strumento militare una vera e propria scuola della Nazione.

Indicativa la vicenda dell'aggettivo Regio nella denominazione dell'esercito unitario. Regio Esercito Italiano per un brevissimo periodo durante le guerre 1859-61 Fanti abolisce ufficialmente sia l'aggettivo che la precedente denominazione di Armata Sarda con la nota n. 76 del 4 maggio 1861 - l'Esercito Italiano torna Regio solo nel 1879, ad opera del ministro della guerra generale Gustavo Mazé de la Roche, fedelissimo di Casa Savoia. Sono gli anni successivi al breve ministero di Carlo Mezzacapo nel primo governo della Sinistra Storica, con i pensionamenti forzati di alti ufficiali piemontesi (tra i quali Raffaele Cadorna) nel 1877 e i timori di un riemergere del dibattito sulla nazione armata. Il ritorno dell'appellativo Regio può essere

${ }^{20}$ Le vicende dell'integrazione delle forze volontarie nell'esercito regio sono ben trattate in MAZZETTI, Massimo, op. cit. 
interpretato sia come un messaggio interno all'istituzione volto a riaffermare la linea della continuità sabaudo-piemontese ${ }^{21}$, sia come una definitiva normalizzazione in senso risolutamente monarchico, palesatasi ormai la non pericolosità di ex-garibaldini e democratici e disinnescata la "rivoluzione parlamentare" del 1876 con il trasformismo. L'ascesa al potere della Sinistra Storica comporta infatti da parte di quest'ultima il rapido e definitivo accantonamento delle alternative all'opzione regia, non solo nel paese, ma anche e soprattutto nelle forze armate. La brevità del ministero Mezzacapo è perfettamente leggibile in questo quadro interpretativo. Del resto, già qualche anno prima, il generale Ricotti ha provveduto ad accantonare le esperienze collaterali di natura compromissoria (guardia nazionale in primis) sostituendole con una vera e propria ideologia militare nazionale incentrata sulla figura del Re.

\subsection{Il "contratto" monarchia-esercito}

Riassumendo: tanto l'esercito risponde ad una esigenza di legittimazione da parte della monarchia, quanto la monarchia risponde egregiamente ad un'ansia di legittimazione dell'esercito, in particolare del corpo ufficiali, gran parte dei cui componenti provengono da esperienze tutt'altro che nazionali ${ }^{22}$. Se l'esercito italiano fino alla Grande Guerra è il «meno compromesso d'Europa negli intrighi politici»²3, è per la forte presenza della figura del Re: la sua prerogativa e la conseguente continuità in tema di politica militare impediscono derive boulangiste. Siamo però convinti che la fedeltà alla monarchia del corpo ufficiali, e dell'esercito, sia una variabile mobile, per quanto tendente alla stabilità in senso positivo. La burocratizzazione che interviene nel lungo periodo di pace tra 1866 e 1915, con relative rivendicazioni sociali (soprattutto a fronte dell'antimilitarismo di fine secolo) ed economiche (quello dell'ufficiale povero è un topos ricorrente nella letteratura militare), rendono la fedeltà dell'esercito a Casa Savoia oggetto di una continua contrattazione, nella quale la monarchia impegna una serie di strumenti.

Anzitutto una gestione del corpo ufficiali mirata, soprattutto nei primissimi anni, a garantire l'egemonia numerica di ex sottufficiali e ufficiali provenienti dalla vecchia Armata Sarda, e soprattutto di un forte nucleo di nobiltà piemontese - assolutamente

${ }^{21}$ DE LEONARDIS, Massimo, op. cit., p. 187.

${ }^{22} \mathrm{~A}$ maggior ragione nel caso napoletano, unica realtà preunitaria paragonabile al Regno di Sardegna: la monarchia sabauda sostituisce un'esperienza monarchico-nazionale scomparsa (per quanto meno completa di quella sarda), ponendo rimedio al vuoto valoriale che incombe sull'ufficialità meridionale.

${ }^{23}$ ROCHAT, Giorgio, MASSOBRIO, Giulio, Breve storia dell'esercito italiano dal 1861 al 1943, Torino, Einaudi, 1978, pp. 40-42. 
maggioritaria sul totale degli ufficiali nobili - fra gli alti gradi ${ }^{24}$. Una sorta di mutua assicurazione tra vecchia Armata Sarda e Re della nuova Italia, che permane di poco variata fino alla fine del secolo, e tramonta parzialmente solo con la Grande Guerra. Alla persistenza della nobiltà piemontese è probabilmente da associare l'assenza di fatto di un istituto massicciamente riscontrabile in Austria-Ungheria e Germania: la nobilitazione. Se nell'esercito austro-ungarico hanno diritto ad un titolo nobiliare tutti gli ufficiali «in grado di comprovare trent'anni [quaranta, dopo il 1866] di condotta irreprensibile» ${ }^{25}$, creando una Neuadel militare numerosa e fideizzata - si pensi che un ufficiale di origini contadine può, attraverso questo istituto, mettere al mondo un figlio nobile! - in Italia la nobilitazione di ufficiali (tra l'altro solo di altissimo grado) resta un fatto sporadico.

Una prerogativa regia che viene invece spesa più che volentieri a favore dell'esercito è la nomina senatoriale. Il laticlavio è una sorta di "premio di fine carriera" per alti ufficiali, eccettuati i ministri della Guerra che vi vengono innalzati generalmente all'atto della nomina. Come ha sottolineato Nicola Labanca, i senatori militari, pur aumentando di numero di legislatura in legislatura - nel periodo 1861-1922 sono in tutto 142, secondi solo agli avvocati e ben più numerosi di professori universitari e magistrati - non intervengono attivamente nella vita parlamentare. Anzi, «i militari in questa camera [...] partecipavano sempre meno al dibattito politico della nazione [costituendo] una rappresentanza sociale, piuttosto che politica, la rappresentanza corporativa di un'istituzione - o come anche qualcuno diceva - una rappresentanza del passato, dell'Ancien Régime» ${ }^{26}$.

Anche il fatto che il posto di ministro della Guerra sia sempre ricoperto da un generale (salvo Ricasoli ad interim e Severino Casana in età giolittiana) è una garanzia dell'indipendenza dell'esercito sotto la prerogativa regia, e della continuità nella politica militare - da più parti invocata come necessaria in un paese che si immagina dover preparare (a seconda dei casi) una guerra contro la Francia o contro l'AustriaUngheria. Siamo d'accordo con Rochat e Massobrio ${ }^{27}$ : «non mancarono ministri della guerra imposti dal Re come pegno di continuità delle istituzioni militari [e della prerogativa, diremmo noi], ma neanche ministri scelti dal governo per una svolta precisa nella politica di difesa». Vero, tuttavia salvo Ricotti ${ }^{28}$, Mezzacapo e pochi altri, i

\footnotetext{
24 Cfr. MAZZETTI, Massimo, op. cit.

25 DEAK, Istvan, Gli ufficiali della monarchia asburgica; oltre il nazionalismo, Gorizia, LEG, 2003, pp. 254-255.

${ }^{26}$ LABANCA, Nicola, «Militari deputati e deputati militari (1848-1922)», cit., p. 445.

27 ROCHAT, Giorgio, MASSOBRIO, Giulio, op. cit., p. 43.

28 Protagonista del più clamoroso scontro tra un ministro della Guerra e la Corona, nel 1896.
} 
ministri della Guerra appaiono più "comandati al ministero" come ruolo tecnico che come espressione di una decisa volontà politica in campo militare, del resto spesso assente nel clima trasformista del parlamento liberale e, soprattutto, superata dal patto tra l'ex garibaldino Crispi e la Corona.

Infine, il legame tra Casa di Savoia ed esercito (e Nazione) viene assicurato anche con la diretta partecipazione di re, principi e duchi alle guerre d'indipendenza prima, e con la loro inclusione nel corpo ufficiali poi.

\section{Re soldati, re italiani}

\subsection{Esercito scuola della Nazione e canone familiare}

Secondo la maggior parte degli storici, il bilancio dell'operazione "esercito scuola della Nazione" in Italia è quello di un fallimento, anche per quanto riguarda il corpo ufficiali29. La stessa commissione parlamentare d'inchiesta sull'esercito sostiene del resto, all'inizio del Novecento, che «ciascuna parte d'Italia presenta nell'indole dei suoi abitanti differenze caratteristiche tale che difficilmente comportano identità di metodo nell'educazione, e le brevi ferme non consentono di plasmare un tipo unico di soldato»30. Piero Del Negro giunge alle stesse conclusioni, sottolineando come in Italia ben più forte del binomio militazizzazione-nazionalizzazione sia quello militarizzazione-statalizzazione, intesa quest'ultima come supremazia dell'aspetto burocratico su quello eroico e di conseguenza simbolico ${ }^{31}$. Abbiamo poi visto come la risoluzione traumatica del conflitto tra nazione armata ed esercito regio ponga un grosso freno all'efficacia dello strumento di integrazione militare. Tuttavia, a noi qui interessa cogliere, più che l'efficacia degli strumenti in campo, l'ideologia che essi propongono, perché è su questo piano che la monarchia si gioca la partita.

Anzitutto va detto che, soprattutto nei primi anni dello stato unitario, la gran parte degli ufficiali sente effettivamente pesare sulle proprie spalle quel ruolo di pedagoghi dell'Unità nazionale che andrà poi man mano scemando di fronte agli scarsi risultati

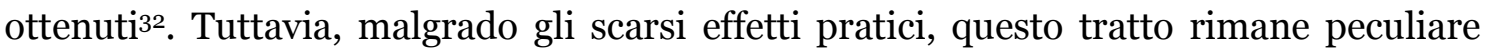

\footnotetext{
29 Cfr. BANTI, Alberto Mario, MONDINI, Marco, op. cit.

$3^{\circ}$ Cit. in DEL NEGRO, Piero, LABANCA, Nicola, STADERINI, Alessandra, op. cit., p. 15.

${ }^{31}$ Ibidem, pp. 15-19.

$3^{2}$ Una testimonianza di tale fervore è data dal dibattito sulla pedagogia militare, studiato da Nicola Labanca. Cfr. LABANCA, Nicola, I programmi dell'educazione morale del soldato; per uno studio sulla pedagogia militare nell'Italia liberale, in Esercito e città dall'Unità agli anni trenta, atti di convegno, Roma, 1989, pp. 521-536.
} 
dell'ideologia auto-giustificativa del corpo ufficiali italiano e del discorso pubblico sulle forze armate - persistenza cui contribuisce una pubblicistica popolare che trova nel romanzo Cuore dell'ex ufficiale De Amicis33 il suo esempio più conosciuto e diffuso.

Domenico Rizzo34 ha recentemente individuato nella riforma del Regolamento di disciplina un altro significativo indicatore della funzione omogeneizzante che in quegli anni si attribuisce all'esercito. Tancredi Fogliani, l'ufficiale incaricato dal ministro Ricotti di stendere materialmente il testo, sovverte la natura dinastica della Disciplina lamarmoriana del 1859, scrivendo un vero e proprio trattato sulla cittadinanza attraverso il servizio militare. Non solo: il regolamento del 1872, rimasto praticamente invariato fino al nuovo secolo, fa esplicitamente riferimento ad un quadro valorialeemozionale di tipo familiare. «I militari di uno stesso corpo devono riguardarsi come membri di una sola famiglia». L'ufficiale è un buon padre-istitutore, che «deve rivolgere tutte le sue cure a sviluppare [...] le forze di mente e di corpo dei suoi subordinati mediante l'istruzione [...]; conoscerne e utilizzare le varie capacità [...]; conciliarsene la stima, il rispetto, e anche l'affezione». Infine, «l'affezione verso il proprio corpo [deve estendersi] agli altri corpi, i quali tutti insieme formano l'esercito»; il quale, attraverso la coscrizione universale, si identifica chiaramente con la Nazione35.

Per i libri di testo scolastici, del resto, «un buon italiano che presta servizio nell'esercito regio è devoto al monarca, suo comandante in capo, e, nella grande famiglia militare, impara lingua e leggi della nuova patria» ${ }^{6}$. Ci troviamo di fronte in definitiva ad una struttura a matrioska, che replica a tutti i propri livelli (famigliareggimento-esercito-Nazione) padri a loro volta figli dei padri di livello superiore, fino al padre di tutti, il Re37. Un canone familiare che sta alla base dell'ideologia autogiustificativa dell'esercito italiano, e non solo.

\subsection{Canone familiare e canone virile: dall'esercito alla Nazione}

Secondo una recente corrente storiografica che ha come esponente più noto Alberto

33 DE AMICIS, Edmondo,Cuore, Milano, Treves, 1886; non a caso lo stesso autore scrive anche ID., La vita militare. Bozzetti, Milano, Treves, 1868; ID., Racconti militari. Libro di lettura ad uso delle scuole dell'esercito, Firenze, Le Monnier, 1869.

34 RIZZO, Domenico, Vita di caserma. Autorità e relazioni nell'esercito italiano del secondo dopoguerra, Roma, Carocci, 2012.

35 Ibidem, pp. 27-29.

${ }^{36}$ BANTI, Alberto Mario, MONDINI, Marco, op. cit., p. 430.

37 Vale la pena di notare che, come già nel giuramento, il Re si sostituisce a Dio come suprema figura paterna; ulteriore riflesso della frattura tra trono e altare, visibile del resto nello stesso Regolamento quando riduce la partecipazione alle funzioni religiose ad una fra le tante prescrizioni morali del militare, accantonando di fatto la centralità del cattolicesimo tipico della Disciplina lamarmoriana; ibidem. 
Mario Banti, la grammatica del discorso nazionale italiano poggia su canoni antichi (famiglia, onore, virtù virile e femminile) reinterpretati e adattati al culto della Nazione ${ }^{8}$. Abbiamo già visto come il canone familiare sia fatto proprio dall'esercito unitario, ma non è una ideologia a puro uso interno dell'istituzione militare.

Nel suo primo discorso della corona, nell'agosto del 1900, il giovane Vittorio Emanuele III rivolge un appello al Parlamento riunito: «Educhiamo le nostre generazioni al culto della Patria, all'onesta operosità, al sentimento dell'onore: a quel sentimento a cui s'ispirano con tanto slancio il Nostro Esercito e la Nostra Armata, che vengono dal popolo e sono pegno di fratellanza, che congiunge nell'Unità e nell'amore della Patria tutta intiera la famiglia italiana»39. Di come le parole di Vittorio Emanuele siano esemplificative di un discorso pubblico iniziato prima dell'Unità, e rimasto nel DNA dell'identità italiana fino ad oggi, ha ben scritto Banti40. Qui ci interessa notare come la famiglia militare sia esplicitamente fusa alla famiglia italiana, trasferendo dall'una all'altra (e viceversa) simboli e rapporti di potere finalizzati in ultima analisi ad un solo obiettivo: la legittimazione di Casa Savoia, figura paterna che in sé riassume l'unità della Patria41.

Il canone familiare è un elemento centrale della saldatura tra monarchia sabauda e Nazione italiana, ma non è l'unico: ugualmente, se non più importante è il canone virile basato sulle gesta guerresche e sugli attributi militari del sovrano.

Quando le sconfitte del 1866 spingono il governo a cercare di marginalizzare re Vittorio Emanuele II nella condotta delle operazioni sul campo, questi accetta ad una condizione: "Il Re ha promesso di lasciar fare e di astenersi da ogni atto che possa disturbare l'opera dei capi militari [...] purché si salvino la convenienza verso di lui dirimpetto all'esercito e alla Nazione perché quando un Re di Prussia ha il comando supremo dell'esercito, il Re d'Italia non può essere da meno»42. L'aveva ben capito Domenico Farini quando scriveva nei suoi diari «La monarchia piemontese, il Piemonte, ebbero l'egemonia in Italia perché dinastia e popolo guerrieri: sfrondate

$3^{8}$ Cfr. BANTI, Alberto Mario, La nazione del Risorgimento. Parentela, santità ed onore alle origini dell'Italia unita, Torino, Einaudi, 2000.

39 Cit. in LABANCA, Nicola, «La morte del padre; militari e regicidio in Italia in una prospettiva comparata», in Cheiron: materiali e strumenti di aggiornamento storiografico, 35-36, 2001, pp. 17-42, p. 33.

${ }_{40}$ Cfr. BANTI, Alberto Mario, Sublime madre nostra; la nazione italiana dal Risorgimento al fascismo, Roma-Bari, Laterza, 2011; BANTI, Alberto Mario, MONDINI, Marco, op. cit.

${ }^{41}$ Oltre al canone familiare, anche il concetto di onore è profondamente insito nell'ideologia auto-giustificativa dell'istituzione militare, e soprattutto del corpo ufficiali (cfr. le magistrali pagine di DEAK, Istvan, op. cit.) per il caso austro-ungarico, in gran parte valide anche per quello italiano; e anche qui non è un caso che lo stesso concetto di onore sia tra quelli elencati da Banti, cfr. BANTI, Alberto Mario, La nazione del Risorgimento, cit.

${ }^{42}$ La frase è del barone Ricasoli, cit. in DE LEONARDIS, Massimo, op. cit., p. 183. 
questa palma e torrete alla monarchia la sola sua ragion d'essere»43.

Come scrivono Banti e Mondini, «la costruzione del mito di Vittorio Emanuele II come soldato dellindipendenza nazionale, nelle due facce dell'eroismo personale e della manifestazione dell'identità guerriera di Casa Savoia, fu [rispetto a quello di Garibaldi, popolare e immediato] un'operazione non solo più tarda (benché sviluppatasi almeno dallo sdegnoso rifiuto a Radetzky), ma soprattutto più consapevole e ricercata dall'alto»44. Costruzione che si attua attraverso la «vasta produzione agiografica e celebrativa» all'indomani della morte del sovrano, e che coinvolge tanto gli antenati quanto i successori del primo re d'Italia. Anche perché alla rilettura in chiave nazionale della storia dinastica attuata dagli storici e dai pubblicisti si affianca un impegno in prima persona della casa reale.

Tutti i Savoia infatti, come del resto accadeva e accade in quasi tutte le dinastie reali, svolgono parte del proprio apprendistato o passano tutta la vita in qualità di ufficiali delle forze armate ${ }^{45}$. Per un Carlo Alberto in prima linea a Goito e Novara, ci sono un giovane Vittorio Emanuele e il di lui fratello generali di divisione; per un re Vittorio Emanuele comandante a San Martino e sul campo a Custoza, ci sono i principi Umberto e Amedeo: il primo protagonista dell'episodio del "quadrato di Villafranca" oggetto di innumerevoli libelli, poesie e rappresentazioni pittoriche; il secondo addirittura ferito da una pallottola austriaca. Nelle due guerre mondiali poi, si mettono in mostra anche i rappresentanti dei rami Aosta e Genova - con Emanuele Filiberto d'Aosta, cugino di Vittorio Emanuele III, protagonista nel primo dopoguerra di un possibile cambio dinastico proprio in virtù della sua celebrità bellica. Tutti i principi, inoltre, sono educati militarmente - celebre il rapporto di odio-amore tra Vittorio Emanuele III, il meno naturalmente portato tra i Savoia per un ruolo guerresco, e il suo rigido precettore generale Osio. Del resto pare che lo stesso Vittorio Emanuele II affermasse: «I miei figli sono soldati e devono battersi», intuendo forse come proprio il canone virile costituisse un pilastro dell'identificazione monarchia-Nazione.

Sulle gesta guerresche di Casa Savoia si costruisce buona parte dell'apparato iconografico che diffonde l'immagine della monarchia tra il popolo. Sia nelle pubblicazioni scolastiche che in quelle divulgative, che in quelle pedagogiche rivolte ai coscritti ${ }^{4}$, Vittorio Emanuele II è sempre presentato in divisa, come più tardi Umberto, e durante e all'indomani della Grande Guerra Vittorio Emanuele III nella creazione del

43 Cit. in LABANCA, Nicola, «Militari deputati e deputati militari (1848-1922)», p. 444.

44 BANTI, Alberto Mario, MONDINI, Marco, op. cit., p. 429.

45 DE LEONARDIS, Massimo, op. cit., p. 191 et seq.

${ }^{6}$ Cfr. LABANCA, Nicola, I programmi dell'educazione morale del soldato; per uno studio sulla pedagogia militare nell'Italia liberale, cit. 
mito del re soldato. Nella missione di legittimare col mito guerriero dei Savoia la monarchia unitaria, esercito e istruzione, questi due strumenti potenti e pervasivi, agiscono in parallelo.

\subsection{Mostrare al popolo il re guerriero}

Abbiamo detto di come buona parte del canone virile sul quale si modella l'immagine pubblica del sovrano abbia caratteri militari, e di come l'essere fisicamente alla testa dell'esercito in guerra ne sia componente primaria. Nel periodo di sostanziale pace tra 1866 e 1914, questa immagine viene perpetuata attraverso le parate militari.

Le celebrazioni pubbliche dell'Italia unitaria hanno infatti sempre carattere militare, e sono intimamente legate alla Corona. Festa dello statuto, genetliaco del sovrano, ricevimento di capi di stato stranieri, persino le nozze dei membri della famiglia reale (e addirittura le nozze d'argento di Umberto e Margherita) sono occasioni per mostrare al popolo il re guerriero alla testa dell'esercito nazionale.

Come ha scritto Ilaria Porciani ${ }^{47}$, quella dello Statuto è la ricorrenza per eccellenza della nazione italiana, tanto da permanere più o meno invariata - parata militare compresa - nella repubblicana festa del 2 giugno. Vale la pena notare come, festeggiando la Nazione nell'anniversario della Carta concessa dalla Corona, già si fondano esplicitamente le due identità, ma non è tutto. Per indagare più a fondo la connessione che si stabilisce in queste occasioni tra immagine militare, del sovrano e nazionale, ci avvaliamo dei rapporti degli addetti militari (attachés militaires) francesi a Roma.

Il 7 giugno del 1874 l'attaché Jules Lemoyne dedica il suo rapporto alla parata dello Statuto:

À l'occasion de la fête du Statut, c'est à dire de la grande fête nationale du royaume, le Roi a passé ce matin en revue toute la garnison de Rome et la garde nationale de la ville. Il s'y trouvait les 2 régiments de grenadiers, 2 régiments d'infanterie, le $10 \mathrm{e}$ régiment de bersaglieri, le district militaire avec ses volontaires d'un an, un détachement du génie, trois batteries d'artillerie à 4 pièces, et un escadron de cavalerie, le tout représentant un effectif de 6 à 7.000 hommes; plus, trois légions de garde nationale comptant un millier d'hommes environ au total.

La tenue des troupes était très belle et donnait une haute idée de l'armée italienne, le défilé a été fort bien exécuté, au milieu d'une foule sympathique, dont les vives

${ }^{47}$ Cfr. PORCIANI, Ilaria, La festa della nazione. Rappresentazione dello Stato e spazi sociali nell'Italia unita, Bologna, Il Mulino, 1997. 
acclamations contrastaient avec le silence réglementaire de la troupe. [...]

Monsieur le Président du Conseil des Ministres, Minghetti, assistait à la revue, portant l'uniforme de major d'état-major, grade qu'il a eu jadis dans l'armée, et le Collier de l'Annonciade que le Roi lui avait envoyé hier 48 .

Nei rapporti degli attachés, ufficiali interessati a riferire essenzialmente di cose militari, le parate sono percepite essenzialmente come avvenimenti, appunto, militari - tanto da meritare sempre un resoconto specifico, a volte lungo decine di pagine, buona parte del quale dedicato alla descrizione della proprietà di tenuta, ritmo di marcia ed evoluzioni in piazza d'armi delle varie unità coinvolte. Tuttavia, gli stessi ufficiali francesi sono concordi nel giudicare tecnicamente priva di interesse la parata in sé, vuoi per le condizioni del terreno di manovra49, vuoi per la scarsa omogeneità delle unità5o o per altri svariati motivi contingenti. Dunque perché ogni anno dedicare numerosi rapporti all'argomento? Per due motivi, entrambi assai significativi.

Primo, perché è essenzialmente in occasione delle parate (oltre che delle grandi manovre annuali) che gli attachés redigono una sorta di consuntivo sullo stato dell'esercito italiano nel quale, oltre agli aspetti puramente militari, tracciano non a caso (assolutamente non a caso, secondo noi) un ritratto dell'esercito come specchio della Nazione italiana. Abbiamo quindi ogni volta elencati quelli che vengono considerati i difetti del soldato italiano (furberia, approssimazione, regionalismo) ma anche le qualità (obbedienza alla gerarchia - in nessun resoconto si fa cenno ad episodi di insubordinazione o ad atti dimostrativi durante una parata - inventiva, capacità di apprendere velocemente). Difetti e qualità che coincidono con quelli attribuiti al popolo italiano nel suo complesso, e che ben rendono l'identificazione, che anche un osservatore esterno percepisce come tale, tra esercito e Nazione. Del resto, anche nella Francia della Terza Repubblica, orfana di un monarca, è all'esercito che si guarda come garante dell’integrità territoriale e morale della Nazione..$^{11}$

${ }_{48}$ Lemoyne, rapporto del 7 giugno 1874, Service Historique de la Défense (SHD) Chateau de Vincennes, 7 N 1358; gustosa ed estremamente significativa l'immagine di Minghetti in uniforme.

49 In particolare negli anni 1880 , quando la parata si svolge negli stretti "boulevards" tra Quirinale e stazione ferroviaria a causa dei lavori in corso al Castro Pretorio.

50 Durante la parata del 14 marzo, genetliaco di Umberto I, spesso sfilavano reclute che si trovavano ai rispettivi reggimenti da meno di un mese, il che chiaramente non poteva dare indicazioni sul grado di efficienza delle unità.

${ }^{51}$ Significativo come gli attachés degli anni Settanta e Ottanta dell'Ottocento, uno dei quali (De La Haye) certamente monarchico avendo servito in quel ruolo già sotto l'Impero, ripetano più volte nei loro rapporti espressioni di invidia per l'ordine e la disciplina che a loro parere caratterizzerebbero esercito e Nazione italiana di contro ai pericoli che correrebbe quella francese, da quando la democrazia vi ha preso piede; frasi che si rarefanno e poi scompaiono con l'arrivo di ufficiali cresciuti professionalmente sotto la Repubblica, e che guardano con 
Secondo, le parate sono anche barometro del consenso del quale gode la monarchia italiana. Se il 14 marzo 1881 infatti, anche sull'onda dell'emozione per il regicidio in Russia avvenuto il giorno prima, l'attaché Brunet ci dipinge l'accoglienza della folla al sovrano come calorosissima, negli anni 1890 i resoconti che il collega Pinsonnière fa delle diverse parate ci mostrano in proporzione crescente folle fredde, scarsissimi applausi, silenzi pesantissimi ${ }^{2}$. Le uniche occasioni che spezzano questo netto calo di popolarità sono le parate straordinarie, quella per le nozze d'argento della coppia reale nel 1893, e quella per il matrimonio del Principe di Napoli (il futuro Vittorio Emanuele III) con Elena del Montenegro il 27 ottobre 1896. Tuttavia, anche in queste occasioni l'entusiasmo del pubblico pare generato più dalle coreografie delle truppe speciali (soprattutto i bersaglieri, ma anche alpini, cavalleggeri e altri reparti richiamati da fuori Roma53), che da un ritrovato affetto verso le persone dei sovrani. Il che ci indica come proprio alla fine del secolo si incrini seriamente quella catena identitaria monarchiaesercito-nazione della quale abbiamo trattato.

\section{Conclusione: la crisi di fine secolo}

Catherine Brice scrive che la comunione monarchia-nazione entra in crisi con gli stati d'assedio di fine secolo, con il Re-padre che ordina ai soldati di sparare sui fratelliitaliani $^{54}$, ma è anche il rapporto monarchia-esercito a subire duri colpi in quegli stessi anni.

Il canone familiare che regola in parte il rapporto tra sovrano e popolo permea come abbiamo visto anche l'esercito. Di conseguenza non sono solo gli italiani cui si spara addosso, ma sono anche gli stessi soldati e ufficiali che sparano a rimanere segnati da tale macroscopica infrazione. Tanto da mettere in crisi l'identità esercitonazione, nel senso che i militari si sentono ormai la vera Nazione contrapposta a quel popolo che ribellandosi, e gettandosi in parte fra le braccia dei movimenti socialisti, ne è escluso. Anche il canone virile subisce un colpo mortale tra gli altipiani etiopici, con la sconfitta di Adua e il conseguente montare dell'antimilitarismo in Italia. Non è un caso che proprio in seguito alla «catena di eventi Adua-1898-regicidio» (alla quale aggiungiamo i disordini del 1893-1894, primo vero momento di rottura) le riviste militari, assieme voce del corpo ufficiali e principale organo che forma la sua opinione,

occhio ben più critico il vicino d'oltralpe.

$5^{2}$ Pinsonnière, rapporto del 20 marzo 1893, SHD, 7 N 1365.

53 Pinsonnière, rapporto del 30 ottobre 1896, SHD, 7 N 1366.

54 BRICE, Catherine, Monarchie et identité nationale en Italie (1861-190o), Paris, Éditions de l'EHESS, 2012, pp. 119 et seq. 
scrivano più o meno esplicitamente di come l'esercito debba salvare la Nazione da popolo e parlamento55: è un pronunciamiento solo teorico, certo, ma di per sé inconcepibile nell'esercito sardo di Lamarmora o in quello unitario dei Ricotti e dei Mezzacapo, ma anche dei Pelloux.

Provvederà la Grande Guerra ad alimentare, cambiandone radicalmente le coordinate, l'ormai esausto bagaglio simbolico che lega monarchia ed esercito, ed entrambi alla Nazione.

55Per un'analisi approfondita della crisi di fine secolo in questa particolare ottica, cfr. LABANCA, Nicola, «La morte del padre; militari e regicidio in Italia in una prospettiva comparata», cit. 


\section{* L'autore}

Jacopo Lorenzini ha conseguito la Laurea Magistrale in Scienze Storiche con lode presso l'Università di Bologna nel 2012 e, in cotutela, presso l'Université Paris VII - Denis Diderot. Attualmente è dottorando all'Università di Siena, con un progetto di tesi intitolato L'élite militare nella nazione. Il caso italiano in prospettiva comparata 1860-1939. Fa parte del collettivo bolognese di giovani storici «Il Caso S».

URL: < http://www.studistorici.com/progett/autori/\#Lorenzini >

\section{Per citare questo articolo:}

LORENZINI, Jacopo, «I re soldati e la Nazione. L'esercito come strumento di legittimazione della monarchia sabauda 1848-1900», Diacronie. Studi di Storia Contemporanea : Le monarchie nell'età dei nazionalismi, 29/12/2013,

URL: < http://www.studistorici.com/2013/12/29//orenzini_numero_16/ >

Diacronie Studi di Storia Contemporanea www.diacronie.it

Risorsa digitale indipendente a carattere storiografico. Uscita trimestrale. redazione.diacronie@hotmail.it

Comitato di redazione: Marco Abram - Jacopo Bassi - Luca Bufarale - Alessandro Cattunar - Elisa Grandi - Deborah Paci - Fausto Pietrancosta - Matteo Tomasoni - Luca Zuccolo

Diritti: gli articoli di Diacronie. Studi di Storia Contemporanea sono pubblicati sotto licenza Creative Commons 2.5. Possono essere riprodotti a patto di non modificarne i contenuti e di non usarli per fini commerciali. La citazione di estratti è comunque sempre autorizzata, nei limiti previsti dalla legge. 